only immunopositive for one marker. Notably NF200 exclusively detected some epithelial fibres and a group of larger diameter neurons $(\varnothing 3-6 \mu \mathrm{m})$ some of which are consistent with observations of A $\delta$-fibre airway mechanoreceptors.

Conclusions We have described neuronal structures in wholemount preparations of tissue from patients suffering from chronic cough. Notably, we described a population of PGP9.5 negative fibres within the airways. Some of these fibres are morphologically consistent with thinly myelinated A $\delta$-fibres described in animal models as 'cough receptors'. These ongoing studies could help define the plasticity of airway nerves in patients with chronic cough.

\section{P156 OUTCOME AND EXPERIENCE OF LAPROSCOPIC NISSENS FUNDOPLICATION IN ADULT PATIENTS WITH SEVERE ASTHMA AND COUGH WHO HAVE GASTROESOPHAGEAL REFLUX}

doi:10.1136/thoraxjnl-2012-202678.217

S Ejiofor, J Brebner, B Barker, A Mansur. Birmingham Heartlands Hospital-Heart of England NHS trust, Birmingham, England

Introduction Gastroesophageal reflux (GORD) is an important co-morbidity in patients with asthma. ${ }^{1}$ It has a detrimental impact on symptoms with many patients being on oral corticosteroids despite maximal proton pump inhibitor (ppi) therapy. ${ }^{1}$ Fundoplication in selected patients with severe asthma has been reported to improve asthma symptoms especially in children. ${ }^{2}$

Birmingham Heartlands Hospital (BHH) is part of Heart of England NHS trust, one of the largest foundation trusts in UK. The Severe and Brittle Asthma Unit which operates from $\mathrm{BHH}$ has a policy of referring selected patients for Fundoplication.

Method In this prospective study all patients undergoing laparoscopic fundoplication were followed up for 12 months. All patients had GORD symptoms refractory to ppi therapy and proven reflux on oesophageal manometry. Prior to surgery and at 3 months patients symptoms were assessed with a) Mini asthma quality of life questionnaire (mAOLQ) b) Reflux Cough Questionnaire (RCQ) and c) Cough symptom Score (CSS). Lung function and medication were documented at follow up visits. Wilcoxon signed rank test was used in statistical analyses.

Results Between December 2009 and June 201213 patients underwent fundoplication. Patient characteristics are shown below.

Fundoplication significantly improved CSS and RCQ at 3 months from baseline, $p=0.002$ and $p=0.008$ respectively. No significant change was seen in $\mathrm{mAQLO}$ or lung function in asthmatic patients. Conclusions Patients with severe cough and asthmatics with debilitating cough benefit from fundoplication. Asthmatics however have no significant improvement in quality of life scores or pulmonary function in the short term following fundoplication.

\section{References}

1. Sontag et al A Am J Gastroenterol. 2003 May; 98(5):987-99.

2. Rothenburg S et al J Pediatric Surg 2012 Jun; 47(6):1101-4.

Abstract P156 Table 1

\begin{tabular}{lcc}
\hline *Age years & 45.8 & \\
M:F & $2: 11$ & \\
${ }^{*}$ DeMeester Score & 59.7 & \\
Diagnosis & Asthma 11 & Cough 3 \\
* Spirometry & Pre & Post \\
& FEV1 2.2 & FEV1 2.15 \\
& FVC 2.83 & FVC 2.84 \\
CSS & 6.38 & 5.29 \\
RCO & 50.2 & 41.6 \\
mA0LO & 3.15 & 3.52 \\
\hline
\end{tabular}

${ }^{*}$ mean values

\section{P157 THE LANGUAGE OF COUGH: A FOCUS GROUP STUDY IN RESPIRATORY DISEASES}

doi:10.1136/thoraxjnl-2012-202678.218

${ }^{1}$ SV Oliver, ${ }^{2 D}$ Yuill, ${ }^{2} \mathrm{~J}$ Yorke, ${ }^{2} \mathrm{AL}$ Caress, ${ }^{2} \mathrm{JA}$ Smith. 'University Hospital of South Manchester, Manchester, UK; ${ }^{2}$ The University of Manchester, Manchester, UK

Background Little is known about the sensations and triggers that provoke cough in patients, or their preferred language to describe their experiences. We have previously collected a list of descriptors covering the themes of triggers, sensations and secretions, generated from individual interviews in a range of respiratory disorders, with a view to developing a questionnaire.

Aims To take the key descriptors and present these to focus groups diagnosed with chronic cough, COPD, ILD, asthma, and non-CF bronchiectasis.

Methods Five semi-structured focus groups were conducted with 22 participants ( 7 chronic cough, mean age 67.7 years (range $57-80$ ) 4 female; 5 asthma, age 66 years (60-71) 2 female; 4 COPD, age 68 years (66-73) 2 female; 3 ILD, age 70 years (65-74) 0 female; 3 nonCF bronchiectasis, age 67.3 years (53-77) 2 female). Using simple manifest content analysis, a list of descriptors was derived based on the frequency of use during individual interviews. These were presented to the focus groups, asking them to describe what each word meant to them, the image it conjured up and whether they could identify a most appropriate word.

Results All focus groups favoured the terms 'phlegm' over 'sputum' (which was considered too "clinical"), 'irritation' over the word 'tickle' (which was considered too "gentle") and the 'need to cough' over an 'urge to cough'. In contrast, there were distinct differences in some disease groups for example, all groups, apart from COPD, recognised specific foods and eating as triggers of coughing. Similarly, all groups, apart from asthma, identified specific smells and odours as triggers of coughing. Patients with COPD, ILD and non-CF bronchiectasis related to the word 'crackle', but asthmatics only associated this word with "infection" and chronic cough patients did not identify with this terminology.

Conclusion These focus group discussions would suggest that whilst some descriptors of cough are felt to be universally appropriate, other descriptors and triggers appear to discriminate between diagnoses. This study will inform the language and content of a future questionnaire to categorise patients by the sensations and triggers provoking their cough.

\section{P158 COUGH SOUND INTENSITY: THE DEVELOPMENT OF A NOVEL MEASURE OF COUGH SEVERITY}

doi:10.1136/thoraxjnl-2012-202678.219

${ }^{1} \mathrm{KK}$ Lee, ${ }^{2} \mathrm{~S}$ Matos, ${ }^{1} \mathrm{~K}$ Ward, ${ }^{1} \mathrm{E}$ Raywood, ${ }^{3} \mathrm{DH}$ Evans, ${ }^{1} \mathrm{~J}$ Moxham, ${ }^{1} \mathrm{GF}$ Rafferty, ${ }^{1} \mathrm{SS}$ Birring. 'King's College London, London, UK; 'Institute of Electronics and Telematics Engineering (IEETA), University of Aveiro, Aveiro, Portugal; ${ }^{3}$ Department of Medical Physics, Leicester Royal Infirmary, Leicester, UK

Introduction and Objectives Cough sound analysis is currently used for the assessment of cough frequency; it is not known if it could be used to assess cough intensity. We investigated the optimal Cough Sound Intensity (CSI) measure by validating it against peak cough flow rate (PCFR).

Methods 17 (11 female) patients with stable chronic cough and 15 (8 female) healthy subjects underwent simultaneous measurements of peak cough flow rate and cough sound during voluntary coughs using 2 types of microphones. Subjects were asked to cough voluntarily 10 times at 5 incremental thoracic pressures as measured by an oesophageal balloon catheter. Visual feedback was given to patients. A range of CSI parameters were determined from the 
cough sound signal. CSI was calculated for the actual cough sound duration and also for a constant duration (time constant) to assess the potential for automation. The repeatability of CSI and the effect of microphone position were assessed. For each subject, PCFRs and CSI data were normalised to values obtained during maximum voluntary cough (MVC).

Results The correlation between the optimal CSI and peak cough flow rate was best for the free-field microphone; median (IOR) correlation coefficient $0.88(0.79-0.92)$ (Table 1$)$. The median correlation for all cough sound intensity remained strong for males $(r=0.90-0.91)$, females $(r=0.79-0.84)$, patients with chronic cough $(r=0.79-0.82)$ and healthy subjects $(r=0.82-0.89)$. The intraclass correlation coefficient for repeatability was good, $r=0.90, p<0.01$. The CSI degraded when the microphone was positioned further away from the mouth, downwards, but not in any other position. The use of a constant cough sound duration to determine CSI did not impact on the association with PCFR (Table 1).

Conclusion Cough sound intensity correlates strongly with PCFR in voluntary cough and is a repeatable measure. The microphone position needs to be standardised as in this study. Further work is needed to automate the analysis of CSI; our preliminary findings suggest this is possible. The CSI has the potential to be developed into a non-invasive, ambulatory outcome measure of cough severity.

Abstract P158 Table 1 Correlation between cough sound intensity (CSI) and peak cough flow rate

\begin{tabular}{lc}
\hline & $\begin{array}{c}\text { Correlation between cough sound } \\
\text { intensity and peak cough flow rate }\end{array}$ \\
\hline Cough sound intensity from laryngeal microphone & $0.84(0.75-0.90)$ \\
Cough sound intensity from free-field microphone & $0.88(0.79-0.92)$ \\
Time-constant CSI from laryngeal microphone & $0.82(0.77-0.90)$ \\
Time-constant CSI from free-field microphone & $0.87(0.79-0.91)$ \\
\hline
\end{tabular}

Data presented as median (IQR) Spearman correlation

coefficients. All p-values $<0.01$.

\section{P159 VALIDATION OF THE VITALOJAK ${ }^{\text {TM }} 24$ HOUR AMBULATORY COUGH MONITOR}

doi:10.1136/thoraxjnl-2012-202678.220

${ }^{1} \mathrm{~K}$ McGuinness, ${ }^{2} \mathrm{~K}$ Holt, ${ }^{2} \mathrm{R}$ Dockry, ${ }^{2} \mathrm{~J}$ Smith. 'University Hospital South Manchester, Manchester, United Kingdom, Manchester, United Kingdom; '2University of Manchester, Manchester, United Kingdom
Introduction Development of novel treatments for cough and its management are hampered by the lack of well validated objective cough frequency methodologies. Previous validations have been performed over limited time periods or in laboratory conditions not always representative of typical usage. We describe the rigorous validation of a semi-automated 24 hour ambulatory cough monitoring system (Vitalojak; Vitalograph; Buckinghamshire, England) operating in a manner which completely replicates routine practise. Methods In total 10 (4 female) patients (mean age 60.4 years [SD \pm 14.1 ] including 6 chronic cough, 2 asthma, 1 COPD and 1 healthy control underwent full 24 Hour ambulatory monitoring (Vitalo$\left.\mathrm{JAK}^{\mathrm{TM}}\right)$. These recordings were manually counted by trained cough counters who also recorded the time at which each cough occurred. These 24 hour recordings were then compressed using custom designed compression software and the sensitivity to cough and the reduced file times were determined. Importantly in each case we confirmed that cough sounds identified in the compressed files were the same sounds identified by the trained manual cough counters in the full 24 hour recording. We tested the software algorithm using three distinct compression levels (1,2 and 3).

Results All results are presented as median (IOR).

Sensitivities to cough (\%) for compression levels 1, 2 and 3 are $100(100,100), 100(99.53,100)$ and $99.92(99.33,100)$ and for reduced file times (minutes) 65.89 (62.40, 83.07), 43.21 (35.94, 57.23) and $26.30(25.07,46.81)$ respectively (Table 1$)$.

Conclusions The vitaloJAK ${ }^{\mathrm{TM}}$ is a reliable, robust and efficient tool for the objective measurement of cough frequency. Importantly it reduces 24 hour recordings by up to $98 \%$ whilst preserving close to $100 \%$ of recorded cough sounds. This development facilitates efficient and speedy manual cough counting and the level of compression achieved represents significant progress towards fully automated cough monitoring.

\section{P160 INCREASED COUGH INTENSITY IN PATIENTS WITH CHRONIC COUGH}

doi:10.1136/thoraxjnl-2012-202678.221

KK Lee, K Ward, E Raywood, J Moxham, GF Rafferty, SS Birring. King's College London, London, UK

Introduction and objectives Cough frequency is increased in chronic cough (CC) compared to healthy subjects. It is not known if patients with CC cough more intensely. We investigated cough intensity in maximum voluntary cough (MVC) in patients with chronic cough and in healthy controls.

Abstract P159 Table 1 Compressed output file times and sensitivities for each subject at compression levels 1, 2 and 3. Uncompressed file length $=1440$ minutes

\begin{tabular}{lrrrrrr}
\hline \multicolumn{7}{c}{ Output times and sensitivity } \\
\hline Subject & Minutes & sensitivity & Minutes & sensitivity & Minutes & sensitivity \\
\hline 1 & 62.01 & 100.00 & 37.40 & 100.00 & 26.23 & 99.37 \\
2 & 63.56 & 100.00 & 31.08 & 99.44 & 24.98 & 98.33 \\
3 & 128.24 & 100.00 & 93.45 & 100.00 & 63.82 & 100.00 \\
4 & 144.00 & 100.00 & 120.72 & 100.00 & 71.90 & 99.83 \\
5 & 35.13 & 100.00 & 25.08 & 100.00 & 20.83 & 100.00 \\
6 & 63.69 & 100.00 & 42.91 & 99.32 & 22.65 & 99.32 \\
7 & 73.15 & 100.00 & 43.50 & 100.00 & 25.32 & 100.00 \\
8 & 86.38 & 100.00 & 56.53 & 100.00 & 41.23 & 100.00 \\
9 & 68.08 & 100.00 & 57.47 & 99.81 & 48.67 & 100.00 \\
10 & 52.71 & 96.95 & 35.45 & 96.95 & 26.37 & 96.59 \\
Mean & 77.69 & 99.70 & 54.36 & 99.55 & 37.20 & 99.34 \\
Median & 65.89 & 100.00 & 43.21 & 100.00 & 26.30 & 99.92 \\
\hline
\end{tabular}

